\title{
Hybrid Metal-Semiconductor Electron Pump for Quantum Metrology
}

\author{
X. Jehl, ${ }^{1, *}$ B. Voisin, ${ }^{1}$ T. Charron, ${ }^{2}$ P. Clapera,${ }^{1}$ S. Ray, ${ }^{1}$ B. Roche, ${ }^{1}$ M. Sanquer, ${ }^{1}$ \\ S. Djordjevic, ${ }^{2}$ L. Devoille, ${ }^{2}$ R. Wacquez, ${ }^{3}$ and M. Vinet ${ }^{3}$ \\ ${ }^{1}$ SPSMS, UMR-E CEA/UJF-Grenoble 1, INAC, F-38054 Grenoble, France \\ ${ }^{2}$ Laboratoire National d'Essais, F-78197 Trappes, France \\ ${ }^{3}$ CEA, LETI, Minatec Campus, F-38054 Grenoble, France
}

(Received 24 May 2012; revised manuscript received 18 March 2013; published 30 May 2013)

\begin{abstract}
Electron pumps capable of delivering a current higher than $100 \mathrm{pA}$ with sufficient accuracy are likely to become the direct mise en pratique of the possible new quantum definition of the ampere. We present here single-island hybrid metal-semiconductor transistor pumps that combine the simplicity and efficiency of Coulomb blockade in metals with the unsurpassed performances of silicon switches. Robust and simple pumping at $650 \mathrm{MHz}$ and $0.5 \mathrm{~K}$ is demonstrated. The pumped current obtained over a voltage-bias range of $1.4 \mathrm{mV}$ corresponds to a relative deviation of $5 \times 10^{-4}$ from the calculated value, well within the $1.5 \times 10^{-3}$ uncertainty of the measurement setup. Multicharge pumping can be performed. The simple design that is fully integrated into an industrial microelectronics process makes it an ideal candidate for national measurement institutes to realize and share a future quantum ampere.
\end{abstract}

DOI: 10.1103/PhysRevX.3.021012

\section{INTRODUCTION}

Linking measurement units to quantum effects rather than to artefacts is a major achievement of modern science [1]. The electrical base unit of the SI, the ampere, which is defined by the force that acts between two ideal conductors, is not directly realized anymore. Instead, since 1990, electrical metrology has been based on voltage and resistance realized with the Josephson and quantum Hall effects [2]. For the SI to fully benefit from their remarkable accuracy, a full set of quantum electrical units based on $e$ and the Planck constant $h$ could be adopted, where the ampere would be a number of elementary charges $e$ per unit time. Just as the speed of light was fixed when the meter was redefined, the fundamental constants $e$ and $h$ would then be fixed, and the mise en pratique of the new definition of the ampere would be made by using the combination of the Josephson and quantum Hall effects. A single-electron-transistor current standard based on an electron pump driven at a frequency $f$, producing a quantized current $I=N e f$ (where $N$ is an integer) with an amplitude of $100 \mathrm{pA}$ or more, could also be used for the realization of the new ampere. Furthermore, such a current is necessary for the quantum metrological triangle experiment that consists of achieving Ohm's law between the three effects used in quantum electrical metrology: the Josephson effect (voltage), the quantum Hall effect (resistance), and the single-electron tunneling effect (current). It aims to test the possible corrections to the relations that

\footnotetext{
*xavier.jehl@cea.fr
}

Published by the American Physical Society under the terms of the Creative Commons Attribution 3.0 License. Further distribution of this work must maintain attribution to the author(s) and the published article's title, journal citation, and DOI. link $e$ and $h$ to the constants associated with three quantum effects: the Josephson constant $K_{J}$, the von Klitzing constant $R_{K}$, and an estimate of the electron charge [3,4].

The understanding of the Coulomb blockade phenomenon [5] rapidly led to the design of electron pumps and turnstiles with both fixed [6,7] and tunable barriers [8]. While turnstiles require a bias voltage to operate, pumps deliver a dc current, even at zero bias. Metallic pumps with fixed-height oxide tunnel barriers and multiple Coulomb islands reached a relative accuracy in the $10^{-8}$ range, with up to six islands in a series between seven tunnel junctions $[9,10]$. Their complex operation was simplified by using only three junctions while lowering errors due to cotunneling with resistors [11]. Despite these efforts, the level of current remained below $10 \mathrm{pA}(f \approx 60 \mathrm{MHz})$, too small by at least 1 order of magnitude for a practical current source. They are also restricted to very low temperatures (below $50 \mathrm{mK}$ ) and cannot accept a bias voltage without a severe degradation of their accuracy [12]. With silicon technology, we previously demonstrated electron pumping in a device with two coupled islands and fixed barriers [13]. Hybrid normal-superconducting turnstiles [14] (which require a finite bias $V_{\mathrm{d}}$ ) based on fixed barriers are also relatively slow but can be operated in parallel to increase the current [15]. An alternative option that uses optically driven self-assembled quantum dots has also been demonstrated recently [16]. A different pumping scheme revived a strong interest in the field because it reached the desired level of current [17-20] and, recently, an accuracy approaching the ppm level [21]. It uses nonadiabatic effects in the population of a dynamical quantum dot [22]. Operations in series of several such pumps have also been realized, with an integrated charge-sensing scheme [23]. At the same time as metallic pumps with fixed barriers were realized, an experiment was performed that 
also uses conventional Coulomb blockade but on a single semiconducting island connected by two tunable barriers [8]. Using $\pi$ phase shifts on the two barriers, quantized currents were obtained, up to $20 \mathrm{MHz}$. Although this pioneering work focuses on turnstile operation, electron pumping at zero bias was also briefly shown and discussed at the end of the report [8]. Later on, a similar experiment with a silicon device [24] reached $f=1 \mathrm{MHz}$, and more recently the same idea was used but with a $\pi$ shift only, resulting again in turnstile operation [25].

In this work, we clarify this pumping mechanism with a novel type of electron pump that uses industrial silicon technology. We show that the phase difference between the sine waves applied to the two tunable barriers determines the number of electrons pumped per cycle and the direction of the current. Some convincing evidence that this pumping mechanism is at play is the observation of an odd or even behavior, depending on the position of the working point with respect to the Coulomb peaks of the device. Since the pump studied here shows that it is possible to combine conventional Coulomb blockade and high speed, it opens new opportunities for quantum metrology. This article is organized as follows. In Sec. II, the sample technology and main characteristics are presented briefly, together with the results of dc electrical transport, which unveil their electrostatic properties. Section III describes the pumping principle and the experimental results, and Sec. IV focuses on experiments designed to assess the stability and accuracy of the pump.

\section{SAMPLE DESIGN AND dc TRANSPORT}

\section{A. Fully depleted silicon-on-insulator technology}

A difficulty with quantized current sources comes from the necessity to have a system that contains at some point a well-defined number of charges $e$. Whether this quantization relies on a high charging energy $E_{c}=e^{2} / C$ for metallic dots (here, $C$ is the total capacitance of the island) or on the one-particle energy-level spacing in a confinement potential created by gates, it requires the fabrication of a very small structure at the limit of typical academic nanofabrication facilities. Silicon field-effect transistors (FETs), where the gate length, channel width, and thickness are all below $20 \mathrm{~nm}$, are now developed and mass produced by the microelectronics industry. We fabricate our electron pumps with the advanced fully depleted silicon-on-insulator (SOI) technology that just entered production for consumer electronics. Compared to conventional planar bulk technology, it offers a better electrostatic control over a very small channel, since the thin Si film that forms the active area is isolated from the substrate by a buried oxide layer of $\mathrm{SiO}_{2}$, as is shown in Figs. 1(b) and 1(c). This architecture is also very favorable for Coulomb blockade operation [26]. The very reduced volume of the transistor's channel yields a high
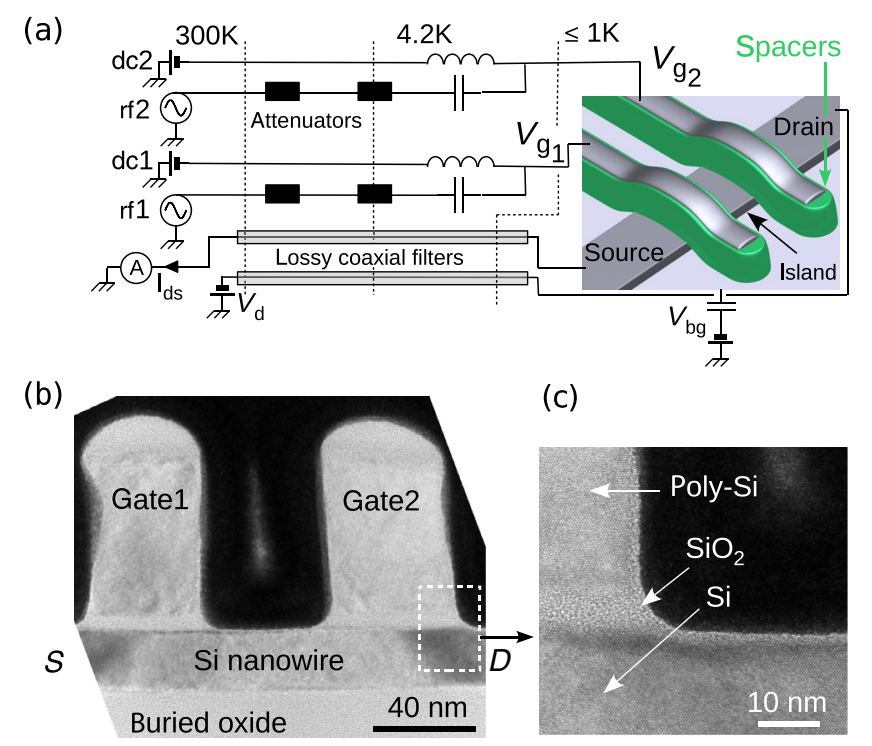

(c)

FIG. 1. (a) Schematic view of the wiring and sample layout. The single metallic island is controlled by two silicon transistors that are controlled by the gate voltages $V_{\mathrm{g} 1}$ and $V_{\mathrm{g} 2}$ on which dc and rf signals are applied. (b),(c) Transmission electron micrographs of the two-gate structure after gate etching. (Spacers as well as silicidation are not shown here.) The single-crystal silicon channel is isolated from the gates by $5 \mathrm{~nm}$ of $\mathrm{SiO}_{2}$. A back-gate voltage $V_{\mathrm{bg}}$ is applied through the 145-nm-thick buried oxide of the silicon-on-insulator wafer.

charging energy, and it is well controlled by the gate electrode. The lever-arm parameter $\alpha=C_{\mathrm{g}} / C$ is close to 1 at $300 \mathrm{~K}$. ( $C_{\mathrm{g}}$ is the gate capacitance.) The fact that it is close to 1 translates into a slope $S$ for the subthreshold current (an important figure of merit for transistors) that approaches the ideal value set by thermal activation of the carriers: $S \approx \ln (10) k_{B} T / e=60 \mathrm{mV} /$ decade at $T=$ $300 \mathrm{~K}\left(k_{B}\right.$ is the Boltzmann constant). In addition to the top gate that acts as a tunable barrier (FET behavior), biasing the substrate with a back-gate voltage $V_{\mathrm{bg}}$ allows us to change both the threshold voltage and the tunnel coupling to source and drain by more than 1 order of magnitude. Thus, the same transistor can be toggled between field-effect and single-electron behaviors [27]. The key steps of fabrication are nanowire lithography and etching, gate-stack deposition $\left(\mathrm{SiO}_{2} / \mathrm{N}+\right.$ doped polysilicon) and patterning, silicon nitride spacer deposition and etch [highlighted in green in Fig. 1(a)], source and drain epitaxy, and doping and silicidation (NiSi). Then, the standard back-end process includes embedding in $\mathrm{SiO}_{2}$, metal lines $(\mathrm{Cu})$, and finally $\mathrm{Al}$ pads. To obtain a pump, two gates are designed in series with a small gap between them, as is shown in Fig. 1. During the silicidation process, the gates and spacers act as a mask. As a result, not only the source and drain become truly metallic but also the island between the two gates. The critical dimensions of the devices measured in this work are given in Table I. 
TABLE I. Dimensions of the samples presented in this article, in $\mathrm{nm}$. $W$ and $T_{\mathrm{Si}}$ stand for the nanowire's width and thickness, $L_{\mathrm{g}}$ is the gate length, $S_{\mathrm{gg}}$ the spacing between the gates, and "Spacers" the spacer length. A single spacer is used for $s 3$, while the standard industrial two-step spacer process is used for $s 1$ and $s 2 . C_{\mathrm{g} 1}$ and $C_{\mathrm{g} 2}$ are the capacitive couplings in $\mathrm{aF}$ between the central island and, respectively, gates 1 and 2 .

\begin{tabular}{lrrrcccc}
\hline \hline Name & $W$ & $T_{\mathrm{Si}}$ & $L_{\mathrm{g}}$ & $S_{\mathrm{gg}}$ & Spacers & $C_{\mathrm{g} 1}$ & $C_{\mathrm{g} 2}$ \\
\hline$s 1$ & 40 & 8 & 30 & 70 & $5+10$ & 19.1 & 20.1 \\
$s 2$ & 60 & 20 & 50 & 50 & $5+10$ & 17.2 & 15.5 \\
$s 3$ & 100 & 25 & 30 & 70 & 15 & 18.4 & 17.2 \\
\hline \hline
\end{tabular}

\section{B. dc electrostatics and transport}

The metallic island is, by design, capacitively coupled to the gates of both transistors; hence, at low temperature, the drain-source current $I_{\mathrm{ds}}$ versus gate voltages $V_{\mathrm{g} 1}$ and $V_{\mathrm{g} 2}$ is a series of antidiagonal lines, as is illustrated in Fig. 2(a) at $0.5 \mathrm{~K}$. Each segment corresponds to the addition of an extra electron on the central island and is limited by the closure of the two FETs. In order to estimate the efficiency of each barrier separately, we set one of them to $+0.8 \mathrm{~V}$ to minimize its contribution to the total current and sweep the other one. Such a measurement is shown in Fig. 2(b). As is expected for industrial FETs, we find a very steep current rise over more than 4 orders of magnitude. At low temperature, the subthreshold slope $S$ is no more set by the ideal thermal activation discussed before but saturates,
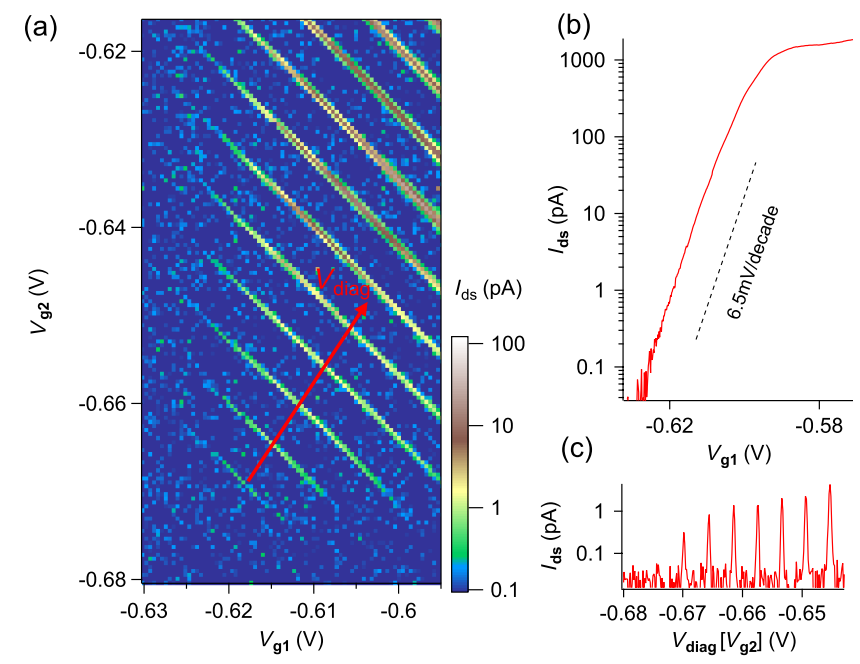

FIG. 2. Drain-source current $I_{\mathrm{ds}}$ for a small drain bias of $50 \mu \mathrm{V}$ at $0.5 \mathrm{~K}$ (sample $s 1$ ). (a) $2 \mathrm{D}$ plot versus both gate voltages, showing the pattern of segments due to the island that is capacitively coupled to both gates. The axis $V_{\text {diag }}$ is defined by passing through the middle of the Coulomb segments. (b) $I_{\mathrm{ds}}$ versus $V_{\mathrm{g} 1}$ for $V_{\mathrm{g} 2}=0.8 \mathrm{~V}$. The steep subthreshold slope over several decades illustrates the quality of the barrier below gate 1. (c) Cut of (a) along $V_{\text {diag }}$. The numerical values correspond to the projection on the $V_{\mathrm{g} 2}$ axis. mainly because the conduction band is broadened by disorder. However, the recorded value of $6.5 \mathrm{mV} /$ decade at $0.5 \mathrm{~K}$ remains excellent and critical for electron pumping. Figure 2(c) shows the periodicity of the current that is recorded when both gates are varied in order to follow the red diagonal line $V_{\text {diag }}$ drawn on top of Fig. 2(a). The values $C_{\mathrm{g} 1}=19.1 \mathrm{aF}$ and $C_{\mathrm{g} 2}=20.1 \mathrm{aF}$ extracted from this graph illustrate the symmetry of the coupling to both FET gates.

\section{ELECTRON PUMPING}

The principle of the pumping experiment is depicted in Figs. 3(a) and 3(b). The white dashed line in Fig. 3(a) corresponds to the region where the dc current measurement falls below the experimental noise floor
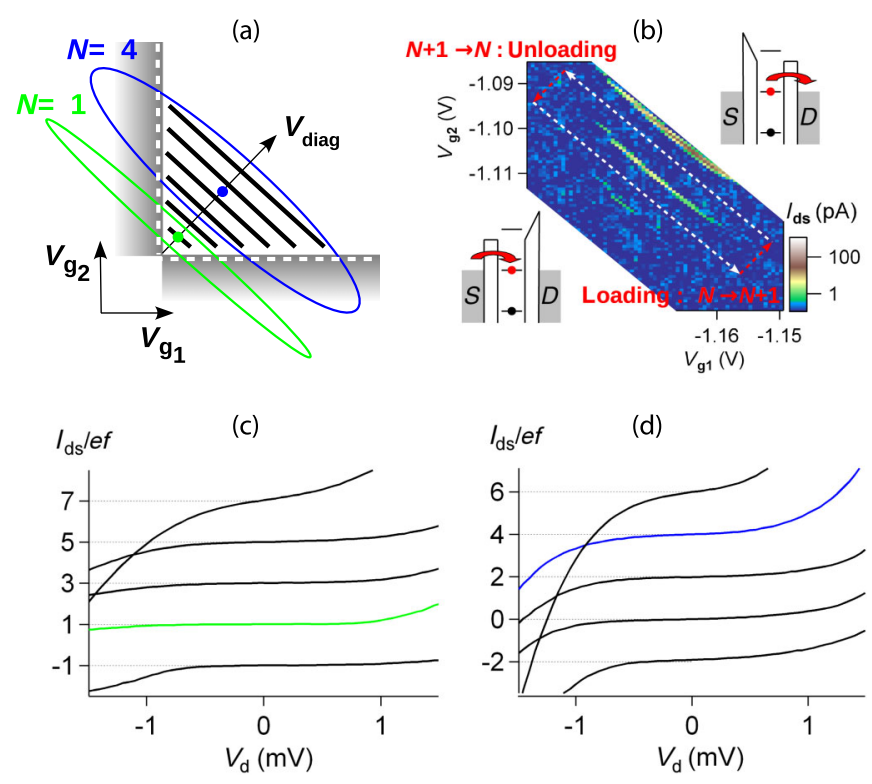

FIG. 3. (a) Schematics of pumping contours realized with phase-shifted rf signals on the two gates. Increasing the shift results in wider ellipses that enclose several Coulomb segments of the island ( 1 and 4 in the examples drawn in green and blue). The white dashed lines and shaded regions indicate the onset of conduction, respectively, through gates 1 and 2. (b) $I_{\mathrm{ds}}$ versus $V_{\mathrm{g} 1}$ and $V_{\mathrm{g} 2}$ in sample $s 2$ at $0.5 \mathrm{~K}$. The dashed lines indicate the four sequences of electron pumping. For the white lines, the potential of the island is kept constant, hence its population as well. The two red ones cross the Coulomb line and therefore require a population change $N \leftrightarrow N+1$. As is sketched, such transitions involving a population change happen when one of the two barriers is very high, forcing electron transfer to occur through the other one. (c) Normalized current $I_{\mathrm{ds}} / e f$ versus drain bias $V_{\mathrm{d}}$ for ellipses at $f=54 \mathrm{MHz}$ centered near a Coulomb line, of equal amplitude on both gates $(-26.5 \mathrm{dBm})$ but of different phase shifts. Plateaus at odd numbers of electrons are found at $1 \mathrm{~K}$, since a growing but always odd number of lines is surrounded as the ellipses get wider. (d) Even plateaus obtained for ellipses centered in between two Coulomb lines and therefore enclosing an even number of lines. 
because the barriers increase rapidly. Application of the phase-shifted sine waves on the gates corresponds to elliptic contours in the $V_{\mathrm{g} 1}-V_{\mathrm{g} 2}$ plane. They can be set to turn around a Coulomb peak without ever crossing a black segment where current flows. It occurs when their center (set by the dc values of $V_{\mathrm{g} 1}$ and $V_{\mathrm{g} 2}$ ) is chosen on or near the Coulomb segment and the extension is large enough, as is shown by the ellipses in Fig. 3(a). This contour has been previously used with silicon but with extremely opaque barriers (always larger than $10^{11} \Omega$ ), resulting in extremely slow $(1 \mathrm{MHz})$ and single-charge pumping[24]. With barriers more than 6 orders of magnitude more transparent, we can pump faster and extend the contour to turn around several Coulomb lines, as is illustrated by the blue ellipse in Fig. 3(a). Instead of a net current $I=e f$ for the green ellipse, one expects $I=N e f$, where $N$ is the number of Coulomb lines enclosed by the contour $(N=4$ for the blue ellipse). The sign of the current is simply changed by turning in the other direction. Although it has not been reported so far, the periodicity of the Coulomb oscillations should yield a different evolution of $N$, depending on the position of the contour center. With ellipses centered near a line, only odd numbers of lines are enclosed, while only even numbers occur for ellipses that are right in between two lines. These two situations have been experimentally realized. The corresponding pumped current plateaus are shown, respectively, in Figs. 3(c) and 3(d). They follow exactly the expected odd or even behavior.

The evolution of the slope of the plateaus as a function of different parameters has been investigated at high frequency. The results for sample $s 2$ are shown in Fig. 4. The evolution of the pumped current versus $V_{\text {diag }}$ is shown in Fig. 4(a) at $0.5 \mathrm{~K}$ for $V_{\mathrm{d}}=0$. The rms amplitudes of the $\mathrm{rf}$ signals at $650 \mathrm{MHz}$ on the gates are 19 and $19.8 \mathrm{mV}$, in order to account for the small difference in couplings to the two gates, and therefore align the long axis of the ellipse with the Coulomb segment pattern. The phase difference between the ac gate voltages is set as $189^{\circ}$, in order to preferentially pump -1 electron. When the ellipse encloses only one Coulomb segment, a quantized plateau at $I_{\mathrm{ds}} /$ ef $=-1$ is obtained. Moving along $V_{\text {diag }}$, there are regions where two segments are partially enclosed. In that case, there is a tendency to pump more than one electron; hence, the recorded current periodically increases. This period (approximately $4.1 \mathrm{mV}$ ) is equal to the one extracted from the conductance measurements from which the gate capacitances given in Table I are obtained. A working point, indicated by the cross in Fig. 4(a), is chosen to study the dependence of the pumped current with bias $V_{\mathrm{d}}$ [Fig. 4(b)] and $\mathrm{rf}$ amplitude [Fig. 4(c)] and phase [Fig. 4(d)]. An important
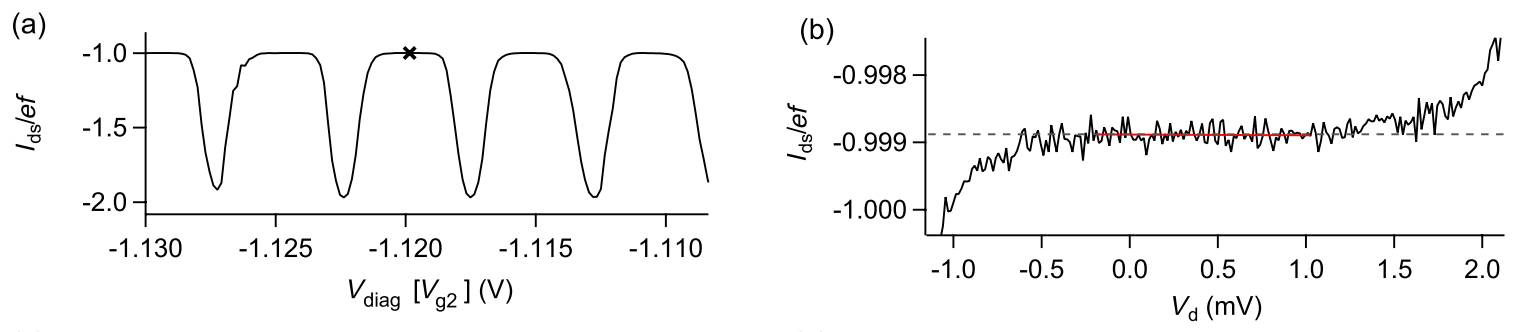

(c)

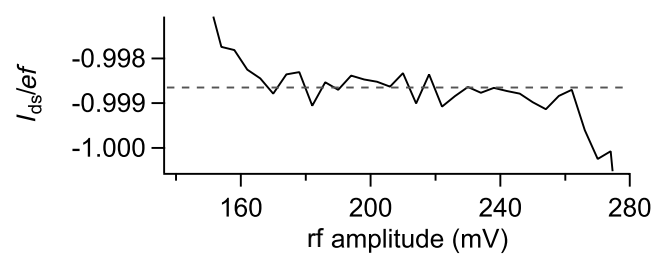

(d)

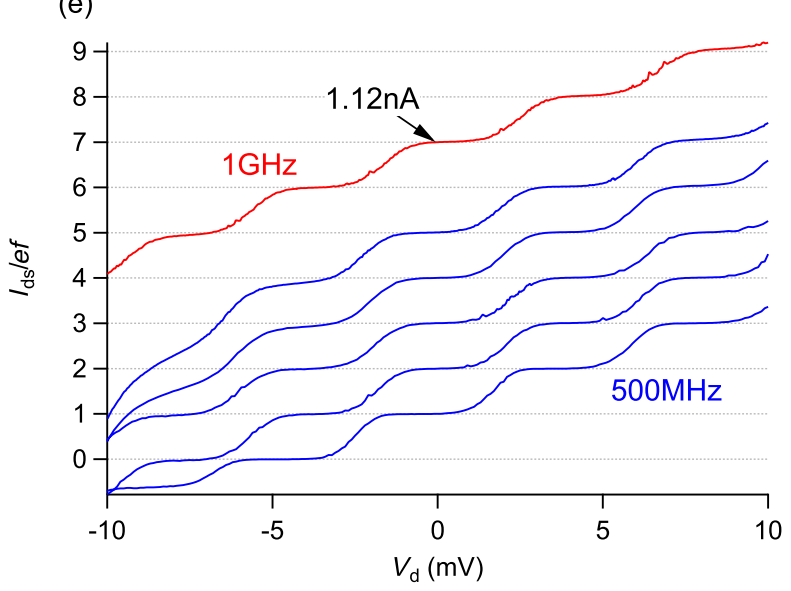

FIG. 4. (a) Pumped current $I_{\mathrm{ds}} /$ ef versus $V_{\mathrm{diag}}$ for sample $s 2$ and a dephasing of $189^{\circ}$ chosen to pump $N=-1$ electron. The cross indicates the working point chosen for the measurements shown in (b)-(d), all of which are also performed at $650 \mathrm{MHz}$ and $0.5 \mathrm{~K}$. (b) Pumped current versus bias $V_{\mathrm{d}}$. The standard deviation of the linear fit across a region wider than $1 \mathrm{mV}$ (in red) sets a lower limit of $250 \mathrm{G} \Omega$ to the resistance. (c),(d) Pumped current versus rf amplitude on both gates and dephasing, showing a wide range of stability for fixed $N=-1$ operation. For (b)-(d), a dashed horizontal line is displayed as a guide to the eyes. (e) Same data as (b) but on sample $s 3$ at $0.6 \mathrm{~K}$, up to $1 \mathrm{GHz}$, and at higher $V_{\mathrm{d}}$. The Coulomb staircase is observed in the pumped current, as an applied $V_{\mathrm{d}}$ that corresponds to the charging energy of the island also forces the population to change. 
figure of merit for a current source is its output resistance, corresponding to the flatness of the plateau in Fig. 4(b). From the uncertainty in a linear fit of the data across a large span of $V_{\mathrm{d}}(1.2 \mathrm{mV})$, in red, we obtain the lower limit of $250 \mathrm{G} \Omega$ for this resistance. The flatness of the plateaus versus rf amplitude and relative phase are important in order to check that the flat region is wide enough to be well within control. The data shown in Figs. 4(c) and 4(d) give a comfortable working range of $70 \mathrm{mV}$ and $6^{\circ}$.

Operation up to $1 \mathrm{GHz}$ and for several values of $N$ is shown in Fig. $4(\mathrm{e})$ at $0.6 \mathrm{~K}$. At $V_{\mathrm{d}}=0$, a plateau is still obtained up to $N=7$, corresponding to a quantized current $I \approx 1.12 \mathrm{nA}$. For this measurement, with large $N$, i.e., a wide ellipse, the rf amplitude has to be increased significantly to maintain a large penetration into the regions shaded in gray in Fig. 3(a), where the island population will eventually change. A maximum rms amplitude of $33.5 \mathrm{mV}$ is used (for $N=7$ at $f=1 \mathrm{GHz}$ ), which is possible at $600 \mathrm{mK}$ but could be challenging at significantly lower temperatures, where heating can occur [25]. It is clear, however, that the flatness quickly degrades with increasing $N$, as a result of the inadequate trajectory looking too much like a circle. For better performance, a custom trajectory performed with an arbitrary waveform generator could be used [21].

While $N$ is determined near $V_{\mathrm{d}}=0$ by the number of Coulomb lines enclosed by the ellipse, it can be changed with a sufficient $V_{\mathrm{d}}$ bias. These steps in $N$ correspond to the Coulomb staircase. In theory, such a staircase is present in any dot; however, the barriers are often strongly affected by the bias and only the first diamond can be observed. In our system, clear plateaus are still found up to $8 \mathrm{mV}$, with a period of approximately $4 \mathrm{mV}$ [Fig. 4(e)], which can be directly interpreted as the charging energy $(4 \mathrm{meV})$, without any lever-arm parameter. This value is in excellent agreement with Coulomb diamond measurements (not shown). This behavior in $V_{\mathrm{d}}$ is characteristic of systems that rely on conventional Coulomb blockade, whether based on GaAs [8], Si [25], or a metal like the ones mentioned here. It is strikingly different from the pumping scheme where the barriers are made more transparent than the quantum of conductance. In that case, the middle island is fully connected to source or drain (hence, there is no Coulomb blockade), and no $V_{\mathrm{d}}$ dependence is found, even up to $200 \mathrm{mV}$ [28]. The possibility to observe clear plateaus with a bias voltage up to twice the charging energy is directly related to the robustness of the barriers in the off state, a figure of merit called drain-induced barrier lowering in microelectronics.

\section{STABILITY AND ACCURACY EXPERIMENTS}

The accuracy of the pumped current has been assessed with alternating current techniques, in order to cancel the offset of the measurement chain.
First, preliminary current measurements that use a cryogenic current comparator have been carried out on a fourth sample, at a reduced frequency of $50 \mathrm{MHz}$, with the cryogenic current comparator in internal feedback mode. Although this scheme does not allow an accurate measurement of the absolute value of the current, it does allow us to investigate the current step flatness and the long-term stability of the device, which is shown in the Supplemental Material [29,30]. The Allan deviation calculated for a 14-hour-long measurement exhibits a $\tau^{-1 / 2}$ variation with the averaging time $\tau$. This variation is a signature of a whitenoise regime over the whole range of measurement time, up to 14 hours (see Figs. 1 and 2 of the Supplemental Material [29]). The statistical uncertainty can therefore be estimated by calculating the experimental deviation of the mean over the whole set of data. It results in a relative statistical uncertainty of three parts in $10^{6}$ on the current value.

Second, calibrated commercial equipments have been used. Figure 5(a) shows the time evolution of the \pm ef currents that are obtained by switching the phase alternatively between $171^{\circ}$ and $189^{\circ}$, at $0.5 \mathrm{~K}, 650 \mathrm{MHz}$, and $V_{\mathrm{d}}=0$. After changing the phase and waiting for $5 \mathrm{~s}$, two points are recorded for each phase, with a 1-s integration time.

In order to evaluate the robustness of the obtained value with bias voltage, this alternating technique is used but on the much shorter time scale of a few tens of minutes, for
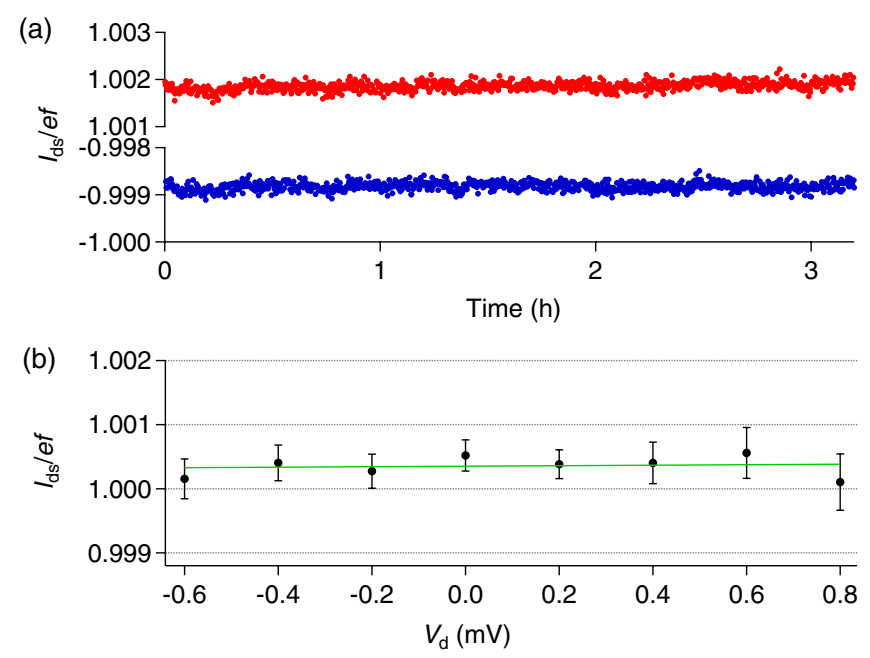

FIG. 5. (a) Alternating pumping experiment performed in sample $s 2$ at $0.5 \mathrm{~K}, 650 \mathrm{MHz}$, and for $V_{\mathrm{d}}=0 \mathrm{mV}$. The dephasing is periodically set to $171^{\circ}$ and $189^{\circ}$, in order to pump, respectively, +1 (red circles) and -1 electron (blue circles) per cycle. This sequence is repeated over several hours. (b) Average values for + and $-e f$ pumping obtained from data similar to (a) but in 10 min only and for several $V_{\mathrm{d}}$. The error bars are twice the statistical standard deviation. This data set yields a value of $I_{\mathrm{ds}} / e f=1.00036$, well within the uncertainty of the measurement setup, and sets a lower limit of $6.8 \times 10^{13} \Omega$ for the resistance (standard deviation of the linear fit shown by the green line). 
which averaging out two-by-two subsequent values for + and $-e f$ is beneficial (see the Supplemental Material [29]). Then, this whole set of data points is averaged to yield a unique value for a certain $V_{\mathrm{d}}$. This final data set is shown in Fig. 5(b). The standard deviation of a linear fit [in green in Fig. 5(b)] across the $1.4 \mathrm{mV}$ of $V_{\mathrm{d}}$ gives a lower limit of $6.8 \times 10^{13} \Omega$ for the resistance. Averaging these seven values yields a final current $I_{\mathrm{ds}} /$ ef $=1.00036$ across a 1.4-mV voltage-bias span, at $650 \mathrm{MHz}$ and $0.5 \mathrm{~K}$, with a standard deviation of 0.00017 . This value is well within the $0.15 \%$ uncertainty in the gain of our measurement setup after calibration.

\section{CONCLUDING REMARKS}

The first experiments presented here on an industrial complementary metal-oxide-semiconductor electron pump have mostly been performed at $650 \mathrm{MHz}$, in order to produce a pumped current of the order of $100 \mathrm{pA}$, high enough to be relevant for the quantum metrological triangle experiment. It should be noted that the experiments were carried out without any radiation shielding around the sample inside the vacuum chamber of a dilution refrigerator. Although our best value falls well below the accuracy of the measurement system, a better shielding could significantly improve further measurements with a more refined setup. The temperature of $0.5 \mathrm{~K}$ at which we did these experiments illustrates the small dimensions (high charging energy) of the nanowire transistors. However, a study in temperature will be needed now, in order to investigate the main cause of errors in the pumping mechanism. An important contribution to these errors may indeed come from the thermal broadening of the Coulomb lines. Another source of errors will arise from the finite steepness of the FET subthreshold slope. Clearly, when the working point is at an extremum of the ellipse, what matters is the ratio of the minimum resistance $R_{\min }$ of one barrier and the maximum resistance $R_{\max }$ reached by the other one [24]. For $R_{\min }$ that is bound to be larger than $10^{5} \Omega$ for Coulomb blockade operation, a resistance of $R_{\max }=10^{12} \Omega$ has to be reached for the other one if one targets a relative uncertainty of $10^{-7}$. This kind of performance is probably reachable at low temperature with the fully depleted SOI technology, which shows unrivaled performance in terms of leakage. A more quantitative study is necessary, using the simulation tools we have developed for our Coulomb blockade devices and single-charge impurities [31]. A realistic simulation of the electrostatic system and tunable FET barriers has recently been implemented and will be compared with experimental data. The reproducibility and robustness of the fabrication technology ensures a consistent quality that allows very exhaustive experimental investigations, since these embedded samples do not age significantly. We have measured the same single-electron-transistor device based on SOI technology 6 yrs apart, and it has shown no significant modification in the pattern of Coulomb oscillations at low temperature. Another advantage of the nanowire transistors is that these pumps operate as FETs at $300 \mathrm{~K}$. The room-temperature measurements provide valuable information on the samples (e.g., maximum conductance, symmetry of the barriers), which allows us to sort and select samples for low-temperature investigation from the thousands available. A practical current standard based on this device can additionally benefit from straightforward integration with silicon technology, which includes on-chip circuits or drive electronics, as well as rf design for a $1-10-\mathrm{GHz}$ operation.

\section{ACKNOWLEDGMENTS}

The authors acknowledge financial support from the French ANR under Project POESI and the EC FP7 FETProactive NanoICT under Project AFSiD. This work was financially supported within the Joint Research Project "Quantum Ampere" within the European Metrology Research Programme (EMRP). The EMRP is jointly funded by the EMRP participating countries within EURAMET and the European Union. The Nanosciences Foundation supports this work. We also thank Mark W. Keller for his comments and careful reading of the manuscript.

[1] J. Flowers, The Route to Atomic and Quantum Standards, Science 306, 1324 (2004).

[2] B. N. Taylor and T. J. Witt, New International Electrical Reference Standards Based on the Josephson and Quantum Hall Effects, Metrologia 26, 47 (1989).

[3] F. Piquemal and G. Genevès, Argument for a Direct Realization of the Quantum Metrological Triangle, Metrologia 37, 207 (2000).

[4] M.W. Keller, Current Status of the Quantum Metrology Triangle, Metrologia 45, 102 (2008).

[5] H. Grabert and M. H. Devoret, Single Charge Tunneling: Coulomb Blockade Phenomena in Nanostructures (Plenum, New York, 1992).

[6] L. J. Geerligs, V.F. Anderegg, P. A. M. Holweg, J.E. Mooij, H. Pothier, D. Esteve, C. Urbina, and M. H. Devoret, Frequency-Locked Turnstile Device for Single Electrons, Phys. Rev. Lett. 64, 2691 (1990).

[7] H. Pothier, P. Lafarge, C. Urbina, D. Esteve, and M. H. Devoret, Single-Electron Pump Based on Charging Effects, Europhys. Lett. 17, 249 (1992).

[8] L.P. Kouwenhoven, A. T. Johnson, N. C. van der Vaart, C. J.P.M. Harmans, and C.T. Foxon, Quantized Current in a Quantum-Dot Turnstile Using Oscillating Tunnel Barriers, Phys. Rev. Lett. 67, 1626 (1991).

[9] M. W. Keller, J. M. Martinis, N. M. Zimmerman, and A. H. Steinbach, Accuracy of Electron Counting Using a 7-Junction Electron Pump, Appl. Phys. Lett. 69, 1804 (1996). 
[10] M. W. Keller, A. L. Eichenberger, J. M. Martinis, and N. M. Zimmerman, A Capacitance Standard Based on Counting Electrons, Science 285, 1706 (1999).

[11] S. V. Lotkhov, S. A. Bogoslovsky, A. B. Zorin, and J. Niemeyer, Operation of a Three-Junction SingleElectron Pump with On-Chip Resistors, Appl. Phys. Lett. 78, 946 (2001).

[12] X. Jehl, M. W. Keller, R. L. Kautz, J. Aumentado, and J.M. Martinis, Counting Errors in a Voltage-Biased Electron Pump, Phys. Rev. B 67, 165331 (2003).

[13] M. Pierre, B. Roche, X. Jehl, R. Wacquez, M. Sanquer, M. Vinet, N. Feltin, and L. Devoille, in Proceedings of the 27th Conference on Precision Electromagnetic Measurements, Daejeon, South Korea, 2010 (IEEE, Piscataway, NJ, 2010), p. 755.

[14] J. P. Pekola, J. J. Vartiainen, M. Möttönen, O.-P. Saira, M. Meschke, and D. V. Averin, Hybrid Single-Electron Transistor as a Source of Quantized Electric Current, Nat. Phys. 4, 120 (2008).

[15] V. F. Maisi, Y. A. Pashkin, S. Kafanov, J.-S. Tsai, and J. P. Pekola, Parallel Pumping of Electrons, New J. Phys. 11, 113057 (2009).

[16] L. Nevou, V. Liverini, P. Friedli, F. Castellano, A. Bismuto, H. Sigg, F. Gramm, E. Müller, and J. Faist, Current Quantization in an Optically Driven Electron Pump Based on Self-Assembled Quantum Dots, Nat. Phys. 7, 423 (2011).

[17] M. D. Blumenthal, B. Kaestner, L. Li, S. Giblin, T. J. B. M. Janssen, M. Pepper, D. Anderson, G. Jones, and D. A. Ritchie, Gigahertz Quantized Charge Pumping, Nat. Phys. 3, 343 (2007).

[18] A. Fujiwara, K. Nishiguchi, and Y. Ono, Nanoampere Charge Pump by Single-Electron Ratchet Using Silicon Nanowire Metal-Oxide-Semiconductor Field-Effect Transistor, Appl. Phys. Lett. 92, 042102 (2008).

[19] B. Kaestner, V. Kashcheyevs, S. Amakawa, M. D. Blumenthal, L. Li, T. J. B. M. Janssen, G. Hein, K. Pierz, T. Weimann, U. Siegner, and H. W. Schumacher, SingleParameter Nonadiabatic Quantized Charge Pumping, Phys. Rev. B 77, 153301 (2008).

[20] S. P. Giblin, S. J. Wright, J. D. Fletcher, M. Kataoka, M. Pepper, T. J. B. M. Janssen, D. A. Ritchie, C. A. Nicoll, D. Anderson, and G. A. C. Jones, An Accurate High-Speed Single-Electron Quantum Dot Pump, New J. Phys. 12, 073013 (2010).
[21] S. P. Giblin, M. Kataoka, J. D. Fletcher, P. See, T. J. B. M. Janssen, J. P. Griffiths, G. A. C. Jones, I. Farrer, and D. A. Ritchie, Towards a Quantum Representation of the Ampere Using Single Electron Pumps, Nat. Commun. 3, 930 (2012).

[22] V. Kashcheyevs and B. Kaestner, Universal Decay Cascade Model for Dynamic Quantum Dot Initialization, Phys. Rev. Lett. 104, 186805 (2010).

[23] L. Fricke, F. Hohls, N. Ubbelohde, B. Kaestner, V. Kashcheyevs, C. Leicht, P. Mirovsky, K. Pierz, H. W. Schumacher, and R. J. Haug, Quantized Current Source with Mesoscopic Feedback, Phys. Rev. B 83, 193306 (2011).

[24] Y. Ono and Y. Takahashi, Electron Pump by a Combined Single-Electron/Field-Effect- Transistor Structure, Appl. Phys. Lett. 82, 1221 (2003).

[25] K. W. Chan, M. Möttönen, A. Kemppinen, N. S. Lai, K. Y. Tan, W. H. Lim, and A. S. Dzurak, Single-Electron Shuttle Based on a Silicon Quantum Dot, Appl. Phys. Lett. 98, 212103 (2011).

[26] M. Hofheinz, X. Jehl, M. Sanquer, G. Molas, M. Vinet, and S. Deleonibus, Simple and Controlled Single Electron Transistor Based on Doping Modulation in Silicon Nanowires, Appl. Phys. Lett. 89, 143504 (2006).

[27] B. Roche, B. Voisin, X. Jehl, R. Wacquez, M. Sanquer, M. Vinet, V. Deshpande, and B. Previtali, A Tunable, Dual Mode Field-Effect or Single Electron Transistor, Appl. Phys. Lett. 100, 032107 (2012).

[28] A. Fujiwara, N. M. Zimmerman, Y. Ono, and Y. Takahashi, Current Quantization Due to Single-Electron Transfer in Si-Wire Charge-Coupled Devices, Appl. Phys. Lett. 84, 1323 (2004).

[29] See Supplemental Material at http://link.aps.org/ supplemental/10.1103/PhysRevX.3.021012 for discussion and data about the long-term stability of the pump evaluated with a cryogenic current comparator.

[30] B. Steck, A. Gonzalez-Cano, N. Feltin, L. Devoille, F. Piquemal, S. Lotkhov, and A.B. Zorin, Characterization and Metrological Investigation of an R-Pump with Driving Frequencies up to $100 \mathrm{MHz}$, Metrologia 45, 482 (2008).

[31] M. Pierre, M. Hofheinz, X. Jehl, M. Sanquer, G. Molas, M. Vinet, and S. Deleonibus, Background Charges and Quantum Effects in Quantum Dots Transport Spectroscopy, Eur. Phys. J. B 70, 475 (2009). 